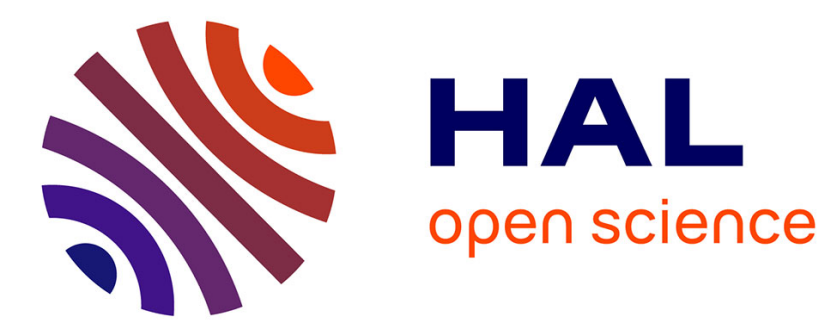

\title{
Using Constraint Propagation for Cooperative UAV Localization from Vision and Ranging
}

Ide-Flore Kenmogne, Vincent Drevelle, Eric Marchand

\section{To cite this version:}

Ide-Flore Kenmogne, Vincent Drevelle, Eric Marchand. Using Constraint Propagation for Cooperative UAV Localization from Vision and Ranging. Decision Making under Constraints, Studies in Systems, Decision and Control, 276, Springer, pp.133-138, 2020, 10.1007/978-3-030-40814-5_16 . hal-02550895

\section{HAL Id: hal-02550895 \\ https://hal.inria.fr/hal-02550895}

Submitted on 22 Apr 2020

HAL is a multi-disciplinary open access archive for the deposit and dissemination of scientific research documents, whether they are published or not. The documents may come from teaching and research institutions in France or abroad, or from public or private research centers.
L'archive ouverte pluridisciplinaire HAL, est destinée au dépôt et à la diffusion de documents scientifiques de niveau recherche, publiés ou non, émanant des établissements d'enseignement et de recherche français ou étrangers, des laboratoires publics ou privés. 


\title{
Using constraint propagation for cooperative UAV localization from vision and ranging
}

\author{
Ide-Flore Kenmogne, Vincent Drevelle, and Eric Marchand \\ Univ Rennes, Inria, CNRS, IRISA \\ ide-flore.kenmogne@inria.fr, \\ \{vincent.drevelle, eric.marchand\}@irisa.fr
}

\begin{abstract}
This paper addresses the problem of cooperative localization in a group of unmanned aerial vehicles (UAV) in a bounded error context. The UAVs are equipped with cameras to tracks landmarks, and a communication and ranging system to cooperate with their neighbours. Measurements are represented by intervals, and constraints are expressed on the robots poses (positions and orientations). Each robot first computes a pose domain using only its sensors measurements, by using set inversion via interval analysis [1]. Then, through position boxes exchange, positions are cooperatively refined by constraint propagation in the group. Results are presented with real robot data, and show position accuracy improvement thanks to cooperation.
\end{abstract}

Keywords: Intervals · Cooperative localization · Constraints propagation

\section{Introduction}

In this paper, we consider the problem of cooperative localization [2] in a group of $N$ unmanned aerial vehicles (UAV). The robots are equipped with cameras, able to see landmarks of known positions. A communication and ranging system provides to each robot $R_{k}$ a means of exchanging data and measuring distances with its neighbours and a base station $B$ (Fig. 1). The goal for each robot is to compute a domain for its pose (position and orientation), assuming bounded error measurements.

The paper is organized as follows: we first present how each robot is able to independently compute a domain for its pose using constraints from camera

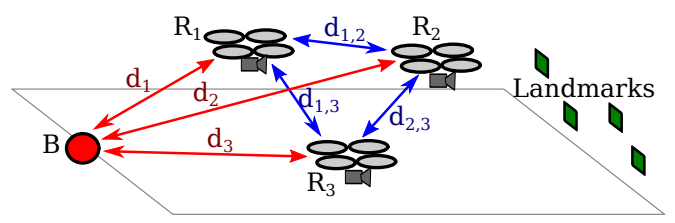

Fig. 1: Cooperative localization with camera and range measurements 
measurements and distance to the base station. Then, in a second part, a cooperative localization method is introduced, in which neighbours positions and distances are introduced as additional constraints to tighten the pose domain of each robot, thanks to data exchange. We finally provide experimental results obtained with quadcopter mini-drones.

\section{Vision-based pose computation}

This section addresses pose estimation [3] from camera measurements. To compute the pose $\mathbf{r}=(x, y, z, \phi, \theta, \psi)$ of a UAV amount in estimating the transformation ${ }^{r} \mathbf{T}_{w}$ between the world reference frame and a reference frame attached to the robot. We assume that the rigid transformation ${ }^{c} \mathbf{T}_{r}$ between the camera and the robot frame is known from calibration [4], and the camera is calibrated.

For a known landmark of $3 \mathrm{D}$ coordinates ${ }^{w} \mathbf{X}$ in the world reference frame, the normalized coordinates $\mathbf{x}=\left({ }^{c} x,{ }^{c} y\right)$ of its projection in the camera frame are given by the pinhole model [5]:

$$
\mathbf{x}=\Pi^{c} \mathbf{T}_{r}{ }^{r} \mathbf{T}_{w}(\mathbf{r})^{w} \mathbf{X}
$$

where $\Pi$ is the perspective projection operator.

For each visible landmark ${ }^{w} \mathbf{X}_{i}(i \in 1 . . m)$, we can derive the following constraints:

$$
C_{i}:\left\{\begin{array}{cl}
\left({ }^{c} X_{i},{ }^{c} Y_{i},{ }^{c} Z_{i}\right)={ }^{c} \mathbf{T}_{r} r \\
{ }^{c} \mathbf{T}_{w}(\mathbf{r}){ }^{w} \mathbf{X}_{i} & \\
{ }^{c} X_{i} & \\
{ }^{c} y_{i}=\frac{{ }^{c} Y_{i}}{c Z_{i}}, & \\
{ }^{c} x_{i} \in\left[{ }^{c} x_{i}\right],{ }^{c} y_{i} \in\left[{ }^{c} y_{i}\right] & \text { bounded error camera measurement } \\
{ }^{c} Z_{i}>0 & \text { front looking camera }
\end{array}\right.
$$

We then define the image-based pose estimation problem as a constraint satisfaction problem (CSP) as:

$$
\mathcal{H}_{\text {img }}:\left(\begin{array}{c}
\mathbf{r} \in[\mathbf{r}], \\
\left\{C_{i}, i \in 1 \ldots m\right\}
\end{array}\right)
$$

This CSP is solved with Contractor Programming [6] and Set Inversion via Interval Analysis (SIVIA) [7]. The altitude $[z]$, pitch $[\theta]$ and roll $[\phi]$ components of the initial domain $[\mathbf{r}]$ are set thanks to onboard sensors (altimeter and inertial measurement unit). An outer approximation of the feasible domain for the pose $\mathbf{r}$ is obtained in the form of a subpaving. An example of solution set for one robot is shown in Fig. 2a (see [8]).

Assuming a bounded error measurement $[d]$ of the distance $d$ between the robot and the base station $B$ is available, it can be used as an additional constraint on the robot position $\mathbf{p}=(x, y, z)$, to get a tighter pose estimate:

$$
d=\|\mathbf{p}-\mathbf{b}\|_{2}, \quad d \in[d]
$$

where $\mathbf{b}$ the known position of the base station. We obtain a tighter domain as presented in Fig. 2b. 


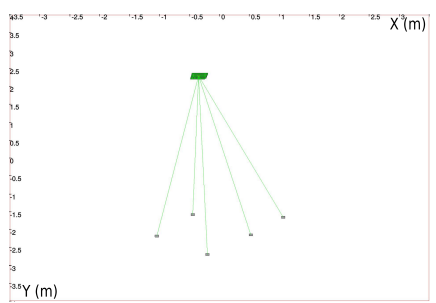

a. using camera only

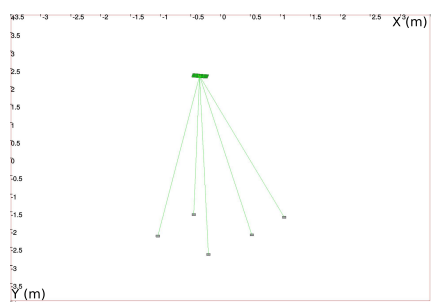

b. using camera and range to base

Fig. 2: Pose domain computation for a single drone (horizontal projection)

Dealing with camera tracking failures The subpaving computed with SIVIA can be empty. This situation corresponds to inconsistencies in the measurements, due to failure of the visual tracking of one or several landmarks (see [8] for more details). In this case, our approach put aside the image measurements and use only the distance to the base station $B$ to compute $\mathbb{S}_{\mathbf{r}_{k}}^{+}$. The solution-set is in this case a ring centered on $B$.

\section{Using range measurements for cooperative localization}

Assuming each UAV is equipped with a communication system with ranging capabilities, each robot therefore measures range to its neighbours and cooperate with them by exchanging its position.

Sharing positions Lets consider one robot $R_{k}(k \in 1 . . N)$ from the group. $\mathcal{N}(k)$ denotes the neighbours of $R_{k}$, i.e. the robots within communication range.

At each time step, $R_{k}$ first computes an outer subpaving $\mathbb{S}_{\mathbf{r}_{k}}^{+}$, that contains all the feasible poses, considering the camera and base distance bounded-error measurements (as presented in Section 2).

Once the pose domain $\mathbb{S}_{\mathbf{r}_{k}}^{+}$is computed, the robot computes the bounding box of its position domain $\left[\mathbf{p}_{k}\right]=\square \operatorname{proj}_{\mathbf{p}} \mathbb{S}_{\mathbf{r}_{k}}^{+}$, where $\square$ is the bounding box operator, and $\operatorname{proj}_{\mathbf{p}}$ is the projection on the position space. $\left[\mathbf{p}_{k}\right]$ is transmitted to all neighboring robots $R_{j}, j \in \mathcal{N}(k)$, and the distances $d_{k, j}$ between $R_{k}$ and $R_{j}$ are simultaneously measured.

Pose contraction At reception of information (position boxes $\left[\mathbf{p}_{j}\right]$ and boundederror distances measurements $\left.\left[d_{k, j}\right]\right)$ from neighboring robots, each robot $R_{k}$ tries to refine its actual pose domain, by propagating the new distance constraints between $R_{k}$ and each of its neighbours. Recalling that $\mathbf{p}_{k}=\left(x_{k}, y_{k}, z_{k}\right)$ is the position of $R_{k}$, each robot $R_{k}$ contracts a local CSP $\mathcal{H}_{k}$ defined as follows:

$$
\mathcal{H}_{k}:\left(\begin{array}{l}
\mathbf{p}_{k} \in \operatorname{proj}_{\mathbf{p}}\left(\mathbb{S}_{\mathbf{r}_{k}}^{+}\right), \\
\mathbf{p}_{j} \in\left[\mathbf{p}_{j}\right], j \in \mathcal{N}(k) \\
d_{k, j} \in\left[d_{k, j}\right], j \in \mathcal{N}(k) \\
d_{k, j}=\left\|\mathbf{p}_{k}-\mathbf{p}_{j}\right\|_{2}, j \in \mathcal{N}(k)
\end{array}\right)
$$


We use interval constraint propagation to solve $\mathcal{H}_{k}$, in order to reduce the pose domain $\mathbb{S}_{\mathbf{r}_{k}}^{+}$.

Fixed-point The constraint network formed by the group of robots contains cycles spanning several robots. The contraction of the local CSPs $\mathcal{H}_{k}$ has to be propagated again through the network to improve the pose domain reduction of each robot. If after solving $\mathcal{H}_{k}$, the robot position bounding box $\left[\mathbf{p}_{k}\right]$ is reduced, then the robot $R_{k}$ retransmits its updated $\left[\mathbf{p}_{k}\right]$ to its neighbourhood. This process is iterated until a fixed-point is reached (no more significant improvement of the robots positions bounding boxes).

\section{Experimental results}

The proposed method has been tested with data acquired on Parrot AR-Drone2 UAV, with 5 landmarks made with AprilTag markers (Fig. 3). Image measurement error bounds are set to $\pm 0.5 \mathrm{px}$ and range measurement error is assumed to be within $\pm 5 \mathrm{~cm}$.

Subpavings obtained with 4 robots in cooperative localization are presented in Fig. 4. Figure 4b clearly shows how cooperative localization reduces the feasible pose domain when some robots cannot clearly see the landmarks, by propagating position information of the neighbours.

Table 1 shows how making more robots cooperate in the fleet improves localization, first by reducing the width of the computed position domain (Table 1a), and also by improving the precision when using the center of the domain as a point estimate (Table 1b). With 4 UAVs, the average horizontal position error is less than $5 \mathrm{~cm}$ for all the drones.

Table 1: Horizontal position results, for 1 to 4 robots in the group

\begin{tabular}{|l|l|l|l|l|}
\hline & $R_{1}$ & $R_{2}$ & $R_{3}$ & $R_{4}$ \\
\hline \hline 1 UAV & 1.29 & & & \\
\hline 2 UAVs & 0.86 & 0.56 & & \\
\hline 3 UAVs & 0.80 & 0.52 & 2.98 & \\
\hline 4 UAVs & 0.33 & 0.37 & 0.78 & 0.61 \\
\hline
\end{tabular}

\begin{tabular}{|l|l|l|l|l|}
\hline & $R_{1}$ & $R_{2}$ & $R_{3}$ & $R_{4}$ \\
\hline \hline 1 UAV & 0.226 & & & \\
\hline 2 UAVs & 0.159 & 0.088 & & \\
\hline 3 UAVs & 0.141 & 0.071 & 1.220 & \\
\hline 4 UAVs & 0.040 & 0.046 & 0.248 & 0.192 \\
\hline
\end{tabular}

Horizontal position domain width $(\mathrm{m})$

Average horizontal position error (m)
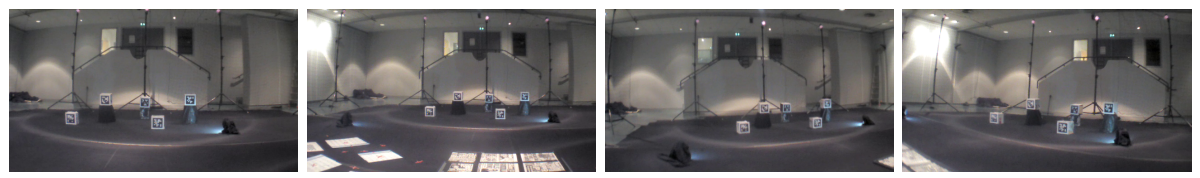

Fig. 3: Onboard cameras views at $t=8 \mathrm{~s}$. Landmarks are boxes with a printed pattern. 


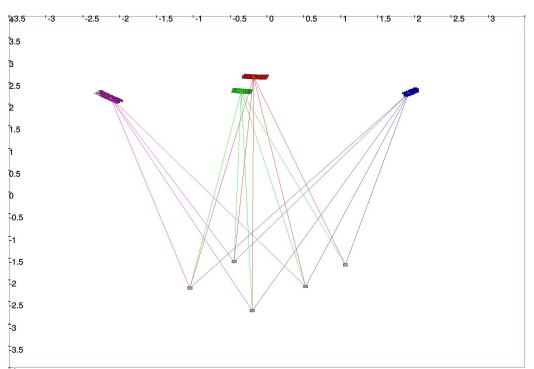

a. $t=0 \mathrm{~s}$, the 4 robots cameras track landmarks

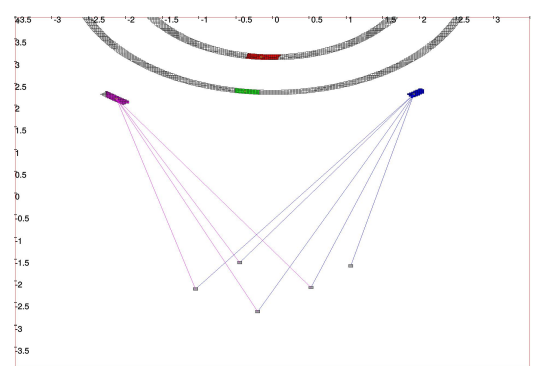

b. $t=6.5 \mathrm{~s}$, the cameras of $R_{1}$ and $R_{2}$ are unable to track landmarks

Fig. 4: Position domains of 4 robots. Black outline: subpavings before communication. Colored domains: subpavings after cooperative localization

\section{Conclusion}

In this paper, we proposed a method to solve cooperative localization in a group of UAVs. Computations rely on interval constraint propagation, assuming bounded error image and distance measurements. Each UAV first independently compute a pose domain from its camera measurements, and then exchange position information with its neighbours to further reduce the position domains of the robots in the group. The method has been applied to real data, and enables to improve the positioning precision of the AUVs thanks to position information propagation. The experiments show that increasing the number of robots in the group provides additional constraints on position, and yields smaller uncertainty of the computed robots poses.

\section{References}

1. R.E. Moore. Interval analysis. Prentice Hall, 1966.

2. S. Roumeliotis, G. Bekey, et al. Distributed multirobot localization. IEEE Trans. on Robotics and Automation, 18(5):781-795, 2002.

3. E. Marchand, H. Uchiyama, and F. Spindler. Pose estimation for augmented reality: a hands-on survey. IEEE Trans. on Visualization and Computer Graphics, 22(12):2633-2651, 2016.

4. R. Tsai. A versatile camera calibration technique for high-accuracy 3D machine vision metrology using off-the-shelf tv cameras and lenses. IEEE Journal on Robotics and Automation, 3(4):323-344, August 1987.

5. Richard Hartley and Andrew Zisserman. Multiple View Geometry in Computer Vision. Cambridge University Press, 2 edition, 2004.

6. G. Chabert and L. Jaulin. Contractor programming. Artificial Intelligence, 173(11):1079 - 1100, 2009.

7. J. Jaulin and E. Walter. Set inversion via interval analysis for nonlinear boundederror estimation. Automatica, 29(4):1053 - 1064, 1993.

8. I. F. Kenmogne, V. Drevelle, and E. Marchand. Image-based uav localization using interval methods. In IEEE/RSJ Int. Conf. on Intelligent Robots and Systems, pages 5285-5291, Sept 2017. 